Case Report

\title{
Mycobacterium peregrinum Pacemaker Pocket Infection: A Case Report and Review of the Literature
}

\author{
A. F. Lazo-Vasquez $(\mathbb{D}$ and J. A. Gonzales-Zamora \\ Division of Infectious Diseases, Department of Medicine, University of Miami, Miller School of Medicine, Miami, Florida, USA \\ Correspondence should be addressed to A. F. Lazo-Vasquez; lazo.alex87@gmail.com
}

Received 7 September 2020; Accepted 29 September 2020; Published 9 October 2020

Academic Editor: Salim Surani

Copyright (c) 2020 A. F. Lazo-Vasquez and J. A. Gonzales-Zamora. This is an open access article distributed under the Creative Commons Attribution License, which permits unrestricted use, distribution, and reproduction in any medium, provided the original work is properly cited.

Mycobacterium peregrinum is a rapidly growing mycobacterium (RGM), subspecies of Mycobacterium fortuitum complex, which can cause infections in the skin, surgical sites, and central lines. It has also been associated with implantable devices such as cardiac devices. Our objective is to present an atypical clinical case of $M$. peregrinum infection associated with a cardiac device, review the published literature, and highlight the importance of this type of RGM infection to enhance their therapeutic success. Only two other cases have been reported of $M$. peregrinum infection associated with cardiac devices. Diagnosis and treatment of $M y$ cobacterium peregrinum infection can be challenging, and the literature is scarce. Better understanding and further research should be conducted regarding this infection.

\section{Introduction}

Nontuberculous mycobacteria (NTM) are environmentally ubiquitous bacteria which have been associated with infections in both immunocompromised and immunocompetent populations [1-3]. Recently, rapidly growing mycobacteria (RGM) have emerged as important human pathogens that can cause a wide range of clinical syndromes in humans, especially $M$. abscessus, M. chelonae, and M. fortuitum [4, 5]. Mycobacterium peregrinum is one of the RGM that belong to the Mycobacterium fortuitum complex and has been rarely reported to infect humans [3]. Overall, the incidence and importance of infections by RGM have been on the rise, and $M$. peregrinum has been associated with central lines, surgical sites, soft tissue, and skin infections [5-7]. Here, we present a case of a permanent pacemaker pocket site infection caused by $M$. peregrinum.

\section{Case Report}

A 59-year-old woman with a history of recent placement of a dual-chamber permanent pacemaker (PPM) due to sick sinus syndrome was admitted to the hospital due to erythema, warmth, and fluctuance over the PPM site (Figure 1). She was at 4 weeks after PPM placement. Her symptoms developed gradually over a course of 5 days. She denied any fever or chills. She did not have any specific exposures over the PPM area other than dressing changes and cleaning with water and soap. There was no recent travel or pet exposure. Her vital signs on admission showed a temperature of $36.5^{\circ} \mathrm{C}$, heart rate of 93 beats per minute, respiratory rate of 18 breaths per minute, and blood pressure of 100/60 mmHg. Physical examination on admission revealed a nondistressed woman, and her chest showed erythema overlying the PPM site with pain to palpation, fluctuance, and evidence of pus from surgical scar (Figure 2). Her laboratory workup showed a white blood cell (WBC) count of $7300 / \mu \mathrm{L}$, with $80.3 \%$ neutrophils, hemoglobin $(\mathrm{Hgb})$ of $12.8 \mathrm{~g} / \mathrm{dL}$, platelet count of $189000 / \mu \mathrm{L}$, lactate of $1.4 \mathrm{mmol} / \mathrm{L}$, glucose of $142 \mathrm{mg} / \mathrm{dL}, \mathrm{BUN}$ of $9 \mathrm{mg} / \mathrm{dL}$, and creatinine of $0.66 \mathrm{mg} / \mathrm{dL}$.

On the day of admission, blood cultures were obtained, and she was started on broad-spectrum antibiotics with vancomycin $1250 \mathrm{mg}$ IV every 12 hours and piperacillin/ tazobactam $4.5 \mathrm{~g}$ IV every 8 hours. She then underwent PPM removal on the $2^{\text {nd }}$ day of hospitalization. Dual-chamber 


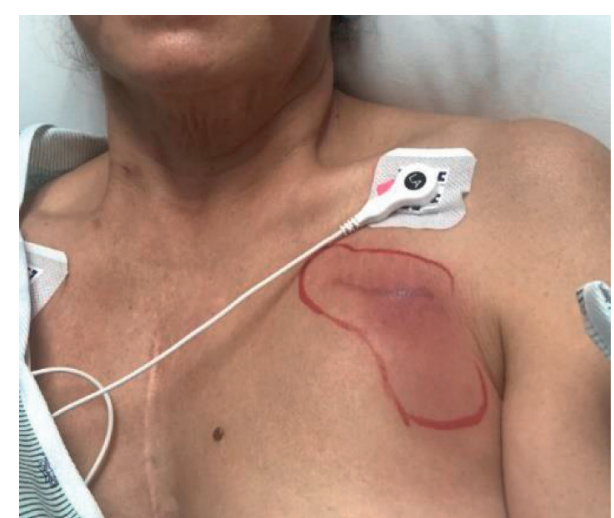

FIGURe 1: Pacemaker pocket site with developing erythema around surgical scar.

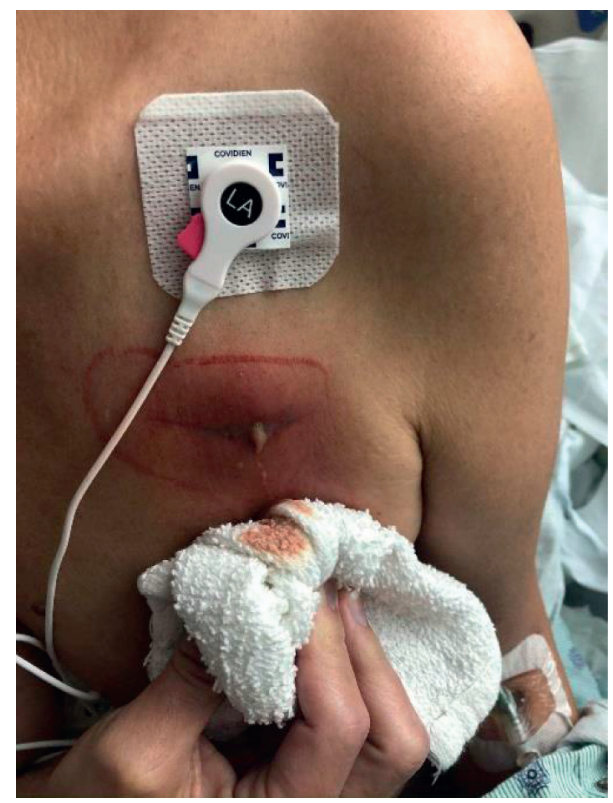

Figure 2: Pacemaker pocket site with purulence expression from the surgical scar.

leads were taken out, and intraoperative specimens from the pocket site were sent to microbiology for stains and cultures. Pathology samples were not sent except for the leads for gross pathological evaluation. No frank pus was obtained intraoperatively, and the leads were removed without any complications. Blood cultures remained negative, and initial gram stain from the intraoperative specimens showed no organisms and rare WBCs. On day 4 of hospitalization, microbiology reported scant growth of acid-fast bacilli from the deep wound cultures, and empiric therapy was changed to amikacin $10 \mathrm{mg} / \mathrm{kg}$ IV daily, imipenem $1 \mathrm{~g}$ IV twice daily, and linezolid $600 \mathrm{mg}$ PO twice daily. The specimen was then sent to the National Jewish Health reference laboratory (Denver, Colorado, USA) for identification by rpoB gene sequencing, which showed Mycobacterium peregrinum.

The patient showed clinical improvement and was discharged from the hospital on day 5 with the proposed empiric intravenous regimen. At 3 weeks of follow-up, the patient reported no side effects from the antibiotics prescribed and had proper healing of the PPM pocket site. Full susceptibilities by broth microtiter dilution methodology were made available (Table 1), and she was switched to moxifloxacin $400 \mathrm{mg}$ PO daily and trimethoprim/sulfamethoxazole $160 / 800 \mathrm{mg}$ twice daily to complete a 4 -month regimen. Of note, the erm(39) gene was not tested for this case, and it was not preincubated in subinhibitory concentrations of macrolide to check for inducible resistance.

The patient was seen at 2 months and completed therapy at 4 months with proper healing of the PPM pocket site (Figure 3). She had no side effects from the antibiotics and did not require reimplantation of the PPM.

\section{Discussion}

Mycobacterium peregrinum is an RGM found ubiquitously in the environment that belongs to the Mycobacterium fortuitum group [1-3]. The clinical presentation of skin and soft tissue infections from RGM usually manifest as localized cellulitis, abscess, sinus tracts, nodules, or chronic ulcers $[8,9]$. It has been postulated that these organisms may preferentially colonize the anterior chest wall since most documented human infections involve the anterior chest such as tunneled catheter infections, sternotomy wound infections, and infections after augmentation mammoplasty procedures [8, 10-12].

There have been several published cases related to M. peregrinum causing infections such as surgical site, cardiac devices, soft tissue, and central catheters [3-7, 13-15]. To our knowledge, there have only been three reported cases of $M$. peregrinum infection associated with cardiac devices, including our patient. One case of $M$. peregrinum infection of an automatic implantable cardioverter-defibrillator was described by Short et al. [12] and another case of a pacemaker lead infection by Amraoui et al. [16]. The case by Short et al. described a similar presentation of $M$. peregrinum infection of a recently placed cardiac device. The patient was treated with a regimen of ciprofloxacin and clarithromycin for a total of 6 weeks. The case by Short et al. involved an $M$. peregrinum infection of the leads of a PPM and associated bacteremia, and he was treated with clarithromycin and ciprofloxacin for a few months and cure was achieved only after removal of the device.

Regarding treatment, RGM have a distinctive antimicrobial susceptibility profile depending on the species, and initial antibiotic treatment should be tailored to this. One must also take into consideration the presence of erm genes which confers resistance to macrolides [17]. Most $M$. fortuitum isolates have an erm gene that could be active or inducible, and macrolides should be used with caution [18]. Studies testing for $\operatorname{erm}(38)$ and erm(39) resistance genes showed that the subspecies $M$. peregrinum does not contain them, but it can still have variable MICs to macrolides due to other mechanisms [19].

For definite treatment, identifying the organism to the species level is paramount to ensure proper antibiotic selection necessary to overcome this infection [6]. Removal of 


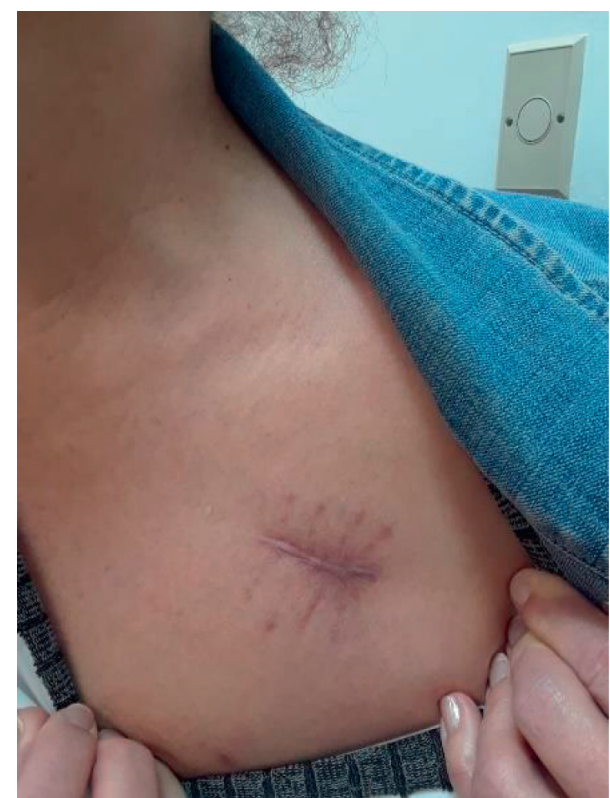

Figure 3: Pacemaker pocket site 2 months after the removal of pacemaker and antibiotic therapy.

TABLE 1: Antibiotic susceptibility and interpretation of M. peregrinum isolates.

\begin{tabular}{lcc}
\hline Antibiotics & MIC $(\mathrm{mcg} / \mathrm{mL})$ & Interpretation \\
\hline Amikacin & $\leq 8$ & $S$ \\
Augmentin & $32 / 16$ & TR \\
Azithromycin & $\leq 16$ & TS \\
Cefepime & $>32$ & TR \\
Cefotaxime & $>64$ & TR \\
Cefoxitin & $\leq 16$ & $S$ \\
Ceftriaxone & $>64$ & TR \\
Ciprofloxacin & $\leq 1$ & $S$ \\
Clarithromycin & $\leq 0.25$ & $S$ \\
Clofazimine & $\leq 0.5$ & TS \\
Clofazimine/amikacin & $\leq 0.5 / 2$ & TS \\
Doxycycline & 8 & $R$ \\
Gentamicin & $\leq 2$ & TS \\
Imipenem & $\leq 2$ & $S$ \\
Kanamycin & 64 & TR \\
Linezolid & $\leq 1$ & $S$ \\
Minocycline & $\leq 1$ & TS \\
Moxifloxacin & $\leq 0.5$ & $S$ \\
Tigecycline & $\leq 0.25$ & TS \\
Tobramycin & 16 & $R$ \\
Trimethoprim/ & $\leq 0.5 / 9.5$ & $S$ \\
sulfamethoxazole & & \\
\hline S & &
\end{tabular}

$S=$ susceptible. $I=$ intermediate. $R=$ resistant. $\mathrm{NI}=$ no CLSI interpretive interpretative guidance. TS=tentative interpretation susceptible. $\mathrm{TI}=$ tentative interpretation intermediate. $\mathrm{TR}=$ tentative interpretation resistant.

the infected device is also essential to guarantee the best chance at cure [18]. Treatment guidelines published jointly by the American Thoracic Society and the Infectious Diseases Society of America (ATS/IDSA) are a valuable resource, but treatment should be tailored to the susceptibility profile of the species. M. peregrinum, a subspecies of the
M. fortuitum complex, is usually susceptible to multiple oral antimicrobial agents, including newer macrolides and quinolones, doxycycline, minocycline, and sulfonamides [18]. At least two agents should be utilized and then tailor the therapy to the susceptibility profile once obtained as they did on both cases reviewed here $[12,16]$.

The duration of antibiotic treatment for this specific type of infection is variable. The IDSA guidelines suggest for serious soft tissue infections with M. fortuitum species to treat for at least 4 months [18]. A review of 11 surgical site infections with $M$. peregrinum by Nagao et al. reported a duration ranging from 6 weeks to 4 months [6]. The two cases of $M$. peregrinum infection associated with cardiac devices mentioned here are based on their treatment duration on clinical response and patient tolerability. One case treated the patient for 6 weeks [12]. The other case treated the patient for several months without success until the cardiac device was completely removed, achieving cure [16]. We treated our case for a total of 4 months and obtained excellent outcomes.

\section{Conclusions}

This case highlights the clinical presentation of an NTM infection related to cardiac devices, a complication that is rare and difficult to treat. To our knowledge, this is the third reported case of $M$. peregrinum, a subspecies of $M$. fortuitum group, causing this type of infection. Diagnosis and treatment are challenging, and therefore, having a high degree of suspicion is key. In the case of culture-negative infection of a cardiac device or around the pocket site, physicians should consider the need for extended incubation or dedicated mycobacterial cultures to improve the yield of NTM growth, especially with the rising significance of various NTM infections. Treatment recommendations for this specific site of infection are limited to expert opinion, but guidelines can be extrapolated from soft and skin tissue infections recommendations of $M$. fortuitum. Combination therapy with at least two agents is necessary to avoid resistance and should be given for at least 4 months. Removal of the infected device is recommended and seems to be essential for cure. Further research is required to elaborate a comprehensive and protocolized management strategy for these infections.

\section{Data Availability}

The data used to support the findings of this study are included within the article.

\section{Conflicts of Interest}

The authors declare no conflicts of interest.

\section{References}

[1] M. Gunaydin, K. Yanik, C. Eroglu et al., "Distribution of nontuberculous Mycobacteria strains," Annals of Clinical Microbiology and Antimicrobials, vol. 12, no. 1, p. 33, 2013.

[2] J. van Ingen, M. J. Boeree, P. N. R. Dekhuijzen, and D. van Soolingen, "Environmental sources of rapid growing 
nontuberculous mycobacteria causing disease in humans," Clinical Microbiology and Infection, vol. 15, no. 10, pp. 888893, 2009.

[3] T. Sakai, C. Kobayashi, and M. Shinohara, "Mycobacterium peregrinum infection in a patient with AIDS," Internal Medicine, vol. 44, no. 3, pp. 266-269, 2005.

[4] J. B. Kalita, H. Rahman, and K. C. Baruah, "Delayed postoperative wound infections due to non-tuberculous Mycobacterium," The Indian Journal of Medical Research, vol. 122, no. 6, pp. 535-539, 2005.

[5] K. Kannaiyan, L. Ragunathan, S. Sakthivel, A. R. Sasidar, Muralidaran, and G. K. Venkatachalam, "Surgical site infections due to rapidly growing mycobacteria in puducherry, India," Journal of Clinical and Diagnostic Research: JCDR, vol. 9, no. 3, pp. DC5-DC8, 2015.

[6] M. Nagao, M. Sonobe, T. Bando et al., "Surgical site infection due to Mycobacterium peregrinum: a case report and literature review," International Journal of Infectious Diseases, vol. 13, no. 2, pp. 209-211, 2009.

[7] P. A. Wachholz, C. S. Sette, D. Caitano do Nascimento, C. T. Soares, S. M. Diório, and P. Y. Masuda, "Mycobacterium peregrinum skin infection: case report," Journal of Cutaneous Medicine and Surgery, vol. 20, no. 3, pp. 249-251, 2016.

[8] F. A. Romero, E. A. Powell, N. E. Babady et al., "Nontuberculous mycobacterial infections after silicone breast implant reconstruction emphasize a diversity of infecting mycobacteria," Open Forum Infectious Diseases, vol. 4, no. 4, Article ID ofx189, 2017.

[9] E. Wolinsky, "Mycobacterial diseases other than tuberculosis," Clinical Infectious Diseases, vol. 15, no. 1, pp. 1-10, 1992.

[10] I. I. Raad, S. Vartivarian, A. Khan, and G. P. Bodey, "Catheterrelated infections caused by the Mycobacterium fortuitum complex: 15 cases and review," Clinical Infectious Diseases, vol. 13, no. 6, pp. 1120-1125, 1991.

[11] G. L. Rodgers, J. E. Mortensen, D. Blecker-Shelly, M. C. Fisher, and S. S. Long, "Two case reports and review of vascular catheter-associated bacteremia caused by nontuberculous Mycobacterium species," The Pediatric Infectious Disease Journal, vol. 15, no. 3, pp. 260-264, 1996.

[12] W. R. Short, C. Emery, M. Bhandary, and J. A. O’Donnell, "Misidentification of Mycobacterium peregrinum, the causal organism of a case of bacteremia and automatic implantable cardioverter defibrillator-associated infection, due to its unusual acid-fast staining characteristics," Journal of Clinical Microbiology, vol. 43, no. 4, pp. 2015-2017, 2005.

[13] G. G. Hogg, M. F. Schinsky, M. M. McNeil, B. A. Lasker, V. A. Silcox, and J. M. Brown, "Central line sepsis in a child due to a previously unidentified Mycobacterium," Journal of Clinical Microbiology, vol. 37, no. 4, pp. 1193-1196, 1999.

[14] J. C. Muthusami, F. L. Vyas, U. Mukundan, M. R. Jesudason, S. Govil, and S. R. B. Jesudason, "Mycobacterium fortuitum: an iatrogenic cause of soft tissue infection in surgery," $A N Z$ Journal of Surgery, vol. 74, no. 8, pp. 662-666, 2004.

[15] M. B. Rodríguez-Gancedo, T. Rodríguez-González, G. Yagüe, P. L. Valero-Guillén, and M. Segovia-Hernández, "Mycobacterium peregrinum bacteremia in an immunocompromised patient with a Hickman catheter," European Journal of Clinical Microbiology and Infectious Diseases, vol. 20, no. 8, pp. 589-590, 2001.

[16] S. Amraoui, J. Texier-Maugein, and P. Bordachar, "PET scan in suspected but unproven pacemaker endocarditis," Archives of Cardiovascular Diseases, vol. 105, no. 2, pp. 125-126, 2012.

[17] K. A. Nash, B. A. Brown-Elliott, and R. J. Wallace Jr, "A novel gene, erm (41), confers inducible macrolide resistance to clinical isolates of Mycobacterium abscessus but is absent from Mycobacterium chelonae," Antimicrobial Agents and Chemotherapy, vol. 53, no. 4, pp. 1367-1376, 2009.

[18] D. E. Griffith, T. Aksamit, B. A. Brown-Elliott et al., "An official ATS/IDSA statement: diagnosis, treatment, and prevention of nontuberculous mycobacterial diseases," American Journal of Respiratory and Critical Care Medicine, vol. 175, no. 4, pp. 367-416, 2007.

[19] K. A. Nash, Y. Zhang, B. A. Brown-Elliott, and R. J Wallace, "Molecular basis of intrinsic macrolide resistance in clinical isolates of Mycobacterium fortuitum," Journal of Antimicrobial Chemotherapy, vol. 55, no. 2, pp. 170-177, 2005. 